\title{
Correction to: Influence of afatinib dose on outcomes of advanced EGFR-mutant NSCLC patients with brain metastases
}

Wan-Ling Tan ${ }^{1}$, Quan Sing Ng${ }^{1}$, Cindy Lim², Eng Huat Tan¹, Chee Keong Toh¹, Mei-Kim Ang ${ }^{1}$, Ravindran Kanesvaran', Amit Jain', Daniel S. W. Tan ${ }^{1,3}$ and Darren Wan-Teck Lim ${ }^{1,4^{*}}$

\section{Correction to: BMC Cancer (2018) 18:1198}

DOI: $10.1186 / \mathrm{s} 12885-018-5110-2$

Following publication of the original article [1], the authors notified us of a typographical error in Table 1.

The corrected Table 1 is presented below.

\section{Author details}

'Division of Medical Oncology, National Cancer Centre Singapore, Singapore, Singapore. ${ }^{2}$ Clinical Trials \& Epidemiological Sciences, National Cancer Centre Singapore, Singapore, Singapore. ${ }^{3}$ Genome Institute of Singapore, A*STAR, Singapore, Singapore. ${ }^{4}$ Institute of Molecular and Cell Biology, A*STAR,

Singapore, Singapore.

Received: 14 December 2018 Accepted: 14 December 2018

Published online: 24 December 2018

\section{Reference}

1. Tan, et al. Influence of afatinib dose on outcomes of advanced EGFR-mutant

NSCLC patients with brain metastases. 2018, 2018;18:1198. https://doi.org/10. 1186/s12885-018-5110-2.

\footnotetext{
* Correspondence: darren.lim.w.t@singhealth.com.sg

${ }^{1}$ Division of Medical Oncology, National Cancer Centre Singapore, Singapore, Singapore
}

${ }^{4}$ Institute of Molecular and Cell Biology, A*STAR, Singapore, Singapore 
Table 1 Patient Baseline Characteristics. The baseline demographics and clinical characteristics of patients with advanced EGFRm + NSCLC treated with first-line afatinib $(n=125)$ in our cohort

\begin{tabular}{ll}
\hline Characteristic & No. \\
\hline Sex & \\
Male & 64 \\
Female & 61 \\
Age at diagnosis, years & \\
Median & 62 \\
Range & $26-86$
\end{tabular}

Ethnicity

Chinese

Malay

Indian

Others

Smoking status

Never

Former

Current

Histotype - NSCLC

Adenocarcinoma

Adenosquamous carcinoma

NOS

EGFR mutation type

\begin{tabular}{|c|c|}
\hline Exon 19 deletion ${ }^{[a]}$ & 87 \\
\hline Exon 21 L858R & 27 \\
\hline Others $^{[\mathrm{b}]}$ & 11 \\
\hline
\end{tabular}

Brain metastases at baseline

$\begin{array}{lll}\text { No } & 82 & 65.6 \\ \text { Yes } & 42 & 33.6 \\ \text { Unknown } & 1 & 0.8\end{array}$

Starting dose of afatinib once daily (OD)

$\begin{array}{lll}40 \mathrm{mg} & 62 & 49.6 \\ 30 \mathrm{mg} & 61 & 48.8 \\ 20 \mathrm{mg} & 1 & 0.8 \\ \text { Unknown } & 1 & 0.8\end{array}$

aE746_A750del; E746_A750delinsIP; E746_A750delinsQP; E746_A750delinsVP; E746_T751delinsV; E746_S752delinsV; E746_P753delinsVS; L747_A750delinsP; L747_T751del; L747_P753delinsS; NOS

bE697Q; A763_Y764insFQEA; Double mutation; Unknown NSCLC Non-small cell lung cancer, NOS Not otherwise specified 